\title{
Aproximaciones de una metodología semiótica para el análisis de la educación en Colombia: un aporte al desarrollo de la edusemiótica
}

\section{Sergio Alejandro Rodríguez Jerez ${ }^{1}$}

1 Miembro de la Edusemiotics Network. Candidato a Doctor en Comunicación y Educación de la Universidad Internacional de la Rioja en España. Magister en Docencia de la Universidad de la Salle. Psicólogo de la Universidad Piloto de Colombia. Director académico de la Asociación Colombiana de Educadores. Docente-Investigador de la Universidad Piloto de Colombia. Docente de Gramática de la Universidad Sergio Arboleda. Director de Desarrollo Humano de la O.N.G. Sembrar Colombia. E-mail: dedofono@hotmail.com

\begin{abstract}
RESUMEN: La aplicabilidad metodológica de la semiótica es una de las grandes preocupaciones y retos de los semiólogos contemporáneos. Por este motivo, el presente artículo tiene como objetivo diseñar un sistema metodológico estructural para el estudio de la educación desde un enfoque semiótico. Esta propuesta es el resultado del trabajo investigativo realizado por la Red de Lectura y Escritura para la Educación Superior (REDLESS) de la Asociación Colombiana de Universidades (ASCUN), mediante el cual se analizó la significación otorgada al concepto de calidad en Colombia. En síntesis el trabajo muestra cómo se puede aplicar la metodología semiótica en la educación, brindando un valioso aporte al desarrollo de la rama edusemiótica presentada en el 12 vo Congreso Mundial de Semiótica en Bulgaria (2014).

Palabras clave: Edusemiotica, Educación, Calidad, Significación, Metodología Semiótica.
\end{abstract}

\section{INTRODUCCIÓN}

En el 12vo Congreso Mundial de Semiótica realizado en Bulgaria en el año 2014, se presentó a la comunidad académica la edusemiótica. La edusemiotica es una rama de la filosofía aplicada en donde se estudia el proceso de significación de los signos en el contexto educativo. Esta rama de la semiótica es liderada por el trabajo de Stables y Semetsky (2014), los cuales han desarrollado grandes aportes para la consolidación de esta nueva propuesta académica que tiene unos antecedentes claramente marcados los cuales se presentarán a continuación.

Como primer antecedente tenemos las ideas propuestas por Kondratov (1973), uno de los lingüistas rusos más representativos, el cual nos plantea lo siguiente:

La semiótica es necesaria para la pedagogía, pues todo el proceso del aprendizaje se reduce a que los hombres aprendan a manejar los signos, ya sea los de la lengua materna o extranjera, ya sea los signos matemáticos, los de la física, de la química o de otras ciencias. (Kondratov, A.M. 1973. p. 31)

Como se puede ver, la necesidad de crear una aplicabilidad metodológica y teórica de la semiótica, en la educación y la pedagogía, ha sido un tema de interés y de necesidad, dado el valioso aporte que ofrece esta disciplina en función del estudio y el entendimiento de las 
configuraciones, producciones e interpretaciones del signo.

Durante el desarrollo de la semiótica han existido, existen y existirán diferentes paradigmas para dar explicación al fenómeno del signo. Estos paradigmas se pueden resumir en dos: los mentalistas y los conductistas. Pese a esta diferencia, tanto los mentalistas como los conductistas llegan a la misma preocupación de crear una rama semiótica que se ocupe del estudio de proceso educativo. Morris (1962), por ejemplo, desde una postura conductista, nos plantea que la semiótica posibilita el mejoramiento del proceso educativo dado que permite el buen uso del lenguaje por parte del educador. Así mismo, Piaget (1978), desde una postura mentalista, plantea la necesidad de tener en cuenta la función semiótica en el proceso del aprendizaje. Los dos anteriores ejemplos son el reflejo de la necesidad de crear la disciplina semiótica encargada de mejorar los procesos educativos en las personas desde el aporte que le puede otorgar la disciplina semiótica.

Lo importante es que pese a las diferencias epistémicas que se tengan, el proceso de significación de los signos de la educación debe articularse en un mismo eje de acción para que no existan incongruencias en el desarrollo de la misma. Esta problemática ya fue solucionada por Jakobson (2012: 37) cuando menciona que: "Cuántas estériles discusiones sobre el mentalismo y el antimentalismo se habrían ahorrado si se abordara la noción del significado en términos de traducción de que ningún mentalista ni ningún conductista podría rechazar".

Con lo anterior se entiende que el proceso semiótico de la educación está en función del proceso de significación que se da mediante la traducción de signos presentes en los seres humanos. Para aclarar lo anterior basta con revisar los aportes de Eco (2013) el cual explica cómo el proceso de traducción de signos se da gracias al sistema de signos presente en el ser humano.

Ampliando lo anterior, Eco (1977) nos revela que el modelo de transformación y entendimiento del signo se genera mediante un proceso en donde el estimulo (signo inicial) pasa por un modelamiento perceptivo, generando una transformación de ese signo inicial para que se logre y se construya el modelamiento semántico. Es decir, que el proceso de significación semiótica, visto como un proceso de traducción de signos, depende del modelamiento perceptivo que tiene aquel que recibe el estímulo. El problema se da cuando el sistema de signos presentes en el receptor no logra configurar la transformación deseada para el modelamiento semántico. Frente a este problema Eco (1989), en su Opera Aperta, plantea, al igual que Barthes, que el proceso de construcción de sentido tiene que ser ilimitado. No obstante, esto genera una serie de dificultades a partir de un concepto fundamental en el entendimiento de los signos el cual es la intencionalidad. Este concepto, muy bien desarrollado por Searle (1999), permite ver que existe un criterio esencial en la transformación del signo el cual debe ser transmitido para cumplir las condiciones de satisfacción del acto del habla. Para Searle $(1999 ; 1994)$ estas condiciones de satisfacción las otorga el propio emisor del signo. Sin embargo, esta postura queda muy reducida y crea dificultades en el entendimiento de la complejidad del signo. Es más, si se asume solo la intención del autor no se tendría presente el sistema de signos del receptor. Por tal motivo, Eco (2013) replantea su hipótesis y manifiesta que el proceso semiótico de interpretación del signo se da 
mediante la interacción de la intencionalidad del autor, la intencionalidad del lector y la intencionalidad operativa. En otras palabras, en el proceso de trasformación y entendimiento del signo están presentes el sistema de signos del autor (emisor), el sistema de signos del lector (receptor) y el sistema de signos del texto (mensaje).

Con lo anterior es necesario aclarar que la edusemiótica posibilita el mejoramiento de los procesos educativos dado que logra integrar los procesos de significación que están presentes en la interacción social. Así mismo, la interacción social, vista desde una perspectiva semiótica, no se reduce, solamente, al intercambio transaccional entre emisor, receptor y mensaje como bien lo aclara Greimas y Courtés (1991). Es decir, que la aplicabilidad metodológica y teórica de la semiótica en la educación permite el mejoramiento de los sistemas del lenguaje y la comunicación gracias al estudio sistémico y funcional del modelamiento semántico teniendo en cuenta los sistemas de signos presentes en las diversas interpretaciones que se presentan en los contextos formativos.

En este orden de ideas, se abren grandes oportunidades para el estudio de los fenómenos mencionados. La intención del presente trabajo es plantear la necesidad de los estudios semióticos en la educación vislumbrando su importancia y ayuda. Por esta razón, en Colombia, se ha venido desarrollando una propuesta que permite utilizar la aplicabilidad metodológica de la semiótica para el mejoramiento de la educación.

Actualmente la Red de Lectura y Escritura de la Asociación Colombiana de Universidades se encuentra trabajando en proyectos investigativos que tienen la intención de mejorar las prácticas de enseñanza a partir del desarrollo de los procesos de lectura y escritura. En este momento, los investigadores de la red están aportando al mejoramiento de la educación superior mediante el análisis de las políticas educativas para el aseguramiento de la calidad a partir de pruebas diseñadas para este propósito (PRUEBAS SABER PRO). Estas pruebas tienen un contexto el cual se presentará a continuación:

El 13 de julio de 2009 se establece en Colombia la ley 1324 "por la cual se fijan parámetros y criterios para organizar el sistema de evaluación de resultados de la calidad de la educación, se dictan normas para el fomento de una cultura de la evaluación, en procura de facilitar la inspección y vigilancia del estado y se transforma el ICFES". Con dicha ley se da inicio a las Pruebas SABER Pro las cuales tienen como objetivo principal evaluar la educación superior a partir de un instrumento estandarizado que permita determinar las competencias de los estudiantes próximos a terminar sus estudios de pregrado. En esta perspectiva, las Pruebas SABER Pro son el resultado de una política educativa que pretender mejorar la calidad de la educación mediante la evaluación sistematizada y estructurada según los criterios establecidos por el Ministerio de Educación Nacional. Este establecimiento de política educativa afectó de manera directa todas las dinámicas educativas del país como se hace evidente en el problema de interconexión entre la política pública y las políticas institucionales.

Como bien lo plantea Gabalán (2007), las pruebas de estado en Colombia, para la Educación Superior, cuentan con un sistema de regulación normativo claro y estructurado desde el gobierno, pero no se complementa con las normativas institucionales. Es decir, que la situación de las políticas para el aseguramiento de la calidad en la educación superi- 
or posee un gran problema de articulación entre el estado y las instituciones educativas.

La falta de articulación se puede observar claramente con el extenso número de investigaciones y trabajos que resaltan la problemática que se da cuando se interpone una visión de estado y la visión institucional en función de lo que es la calidad en educación. De hecho, es la visión capitalista del estado la que se critica por parte de la academia como manipulador de que debería verdaderamente significar la calidad. Por ende, la academia reconoce que no se puede asegurar la calidad con pruebas estandarizadas que se basan en competencias las cuales están en función con la productividad del país.

En este sentido, se tienen dos problemas claramente definidos:

1. No hay articulación entre el estado y las instituciones con relación al establecimiento de las políticas educativas para el aseguramiento de la calidad. Dicha situación se da por la no integración de las políticas estatales con las políticas institucionales.

2. La falta de integración entre la política pública y la política institucional se puede dar por la falta de claridad en el concepto de calidad. Para el estado, este concepto está ligado a una significación atribuida al plano económico, mientras que para un gran número de académicos la calidad es una ideal holístico que no solo depende de la economía.

Finalizando la breve síntesis del problema de educación en Colombia, se puede determinar que éste es causado por un conflicto en la interpretación del significado del concepto de calidad. Dicho conflicto es netamente semiótico, dado que no hay una condición de satisfacción entre el sistema de signos presentes la política pública y las políticas institucionales. Desde una perspectiva semiótica e interpretativa el problema se genera por la multiplicidad de significados que pueden tener las políticas educativas y las políticas institucionales en función del concepto de calidad para el ámbito de la educación.

Tomando como referencia a Magariños de Morentin (2008: 62), el problema de significación al cual estamos haciendo referencia, puede entenderse como un problema "en la producción, comunicación y/o transformación de la significación de algún fenómeno". Teniendo esto claro, se puede deducir que el problema en el establecimiento de las políticas educativas se debe a la falta de integración significante del concepto de calidad. Esta situación causa una divergencia significante, la cual imposibilita una estructuración unificada del objetivo de la educación superior en Colombia en función de la calidad.

Si hacemos una analogía entre el profesor y estudiante, podemos decir que el profesor (es decir la política pública) no logra que los estudiantes (las políticas institucionales) entiendan lo que él entiende por el concepto de calidad. Y así mismo los estudiantes (las políticas institucionales) cuando tienen que decir que es lo que entienden por Calidad dicen algo totalmente diferente a lo que el profesor enseña.

Con esta analogía vemos como la semiótica educativa sirve para analizar los fenómenos propios del proceso de enseñanza en las aulas de clase, y, así mismo, posibilita hacer análisis en función de las políticas y los conceptos que influyen en los diversos ámbitos educativos. Esto se debe a que el proceso de educación se da mediante la apropiación de signos lo cual posibilita la generación del conocimiento.

Culminado con la presentación del problema se hace evidente la necesidad de hacer estudios semióticos en este ámbito con el objetivo de mejorar y solucionar los problemas en 
la educación en Colombia. Teniendo en cuenta este objetivo se viene realizando una investigación con 14 universidades de Colombia en donde se está aplicando una metodológica semiótica para analizar el concepto de calidad en el estado colombiano y las instituciones de educación superior, tomando como referencia la enseñanza de la lectura y la escritura. Esta investigación tuvo como objetivo analizar la importancia de la calidad en Colombia a partir de las políticas y las pruebas estatales que se generaron a partir de la ley 1324, mencionada previamente. Lo que se hizo fue un estudio de estas políticas estatales y las políticas institucionales las cuales tenían relación al desarrollo de la lectura y la escritura en la educación como estrategia única para el mejoramiento en los resultados de las pruebas SABER.

\section{RESULTADOS}

Para llevar a cabo esto se está aplicó una metodología la cual tuvo en cuenta la propuesta desarrollada por Magariños de Morentin (2008: 26) el cual plantea lo siguiente:

Considero, por el contrario, que no existen conceptos que permitan identificar a la semiótica como una entidad autoconsistente en el universo de los fenómenos sociales; la semiótica adquiere, en cada caso, la calidad ontológica del fenómeno cuya significación pretende explicar. Ello es coherente con la posición asumida en el punto anterior, acerca de considerar a la semiótica como una metodología de investigación en ciencias sociales (Magariños de Morentin, 2008: 26)

Según el autor, la semiótica tiene una aplicabilidad pragmática ya que permite establecer un sistema estructurado para la comprensión de los fenómenos. En este sentido, la semiótica utiliza conceptos y operaciones que articulan el proceso de verificación de hipótesis mediante el método científico.

La investigación tuvo como corpus los documentos en donde están establecidas las Políticas de calidad en función de las pruebas SABER PRO. Para ello, se tuvieron en cuenta la Ley 1324 de 2009 y el Decreto 4216 del 30 de octubre de 2009. Así mismo, se recolectaron las políticas institucionales que hicieron referencia a las pruebas SABER PRO. Empero, y debido a la extensión de todos los documentos, se hizo una delimitación de los textos a trabajar mediante una técnica de análisis categorial en donde solo se tuvieron en cuenta los apartados textuales donde se hace referencia explícita del concepto Calidad.

Para ello, se revisaron en todos los documentos el número de veces que aparece el término y se hizo un extracto de los párrafos completos con los cuales se aplicó el análisis semiótico de contraste.

El análisis semiótico de contraste se llevó a cabo mediante un diseño previo de las redes contrastantes las cuales fueron resultado de la normalización, la segmentación, y la definición contextual de cada uno de los textos. Lo que se buscaba con el análisis era determinar la divergencia significante en cada texto con relación al concepto de calidad.

Para lograr esta se establecieron las siguientes operaciones:

1. Operaciones Analíticas: en esta operación se buscaron las redes contrastantes en los 
textos con relación al concepto de calidad en tres etapas:

1.1 Normalización y Segmentación: en esta etapa se depuró el texto en función del material verdaderamente relevante en el análisis semiótico. La etapa fue netamente sintáctica, lo cual permitió un mayor criterio de objetividad con respecto al análisis.

1.2 Definiciones Contextuales: después de la normalización y la segmentación desde la sintáctica, fue necesario realizar un encuadre semántico que otorgó sentido al texto desde un análisis de contexto, lugar y situación.

1.3 Diseño de Redes Contrastantes: en la tercera etapa, y como bien lo plantea Magariños de Morentin (1998: 240), se buscó que "aparezcan diferencias o contradicciones que garanticen que se ha llegado a la identificación de, al menos, más de una Formación Discursiva. La presencia, pues, de la diferencia o de la contradicción (el contenido de tal "diferencia o contradicción" deberá ser tal que provoque la inconsistencia del sistema axiológico y/o conceptual en estudio."

Después de las operaciones analíticas tenemos las operaciones de los límites interpretativos.

2. Operación de los límites interpretativos:

En busca de ser más objetivo con la metodología aplicada, se hizo un análisis interpretativo con relación a la triada de la intencionalidad propuesta por Eco (2013). Como se mencionó en la introducción, existen límites interpretativos los cuales están en función de los tres tipos de intencionalidad: intencionalidad del lector, intencionalidad operativa (o del texto) y la intencionalidad del autor. Debido a esta situación, se hizo una operación semiótica que analizó estas tres intencionalidades después de llevar a cabo las operaciones analíticas.

Como tercera operación, se llevó a cabo la Operación Analítica de Divergencia.

3. Operación analítica de divergencia:

En esta última operación se realizaron inferencias con respecto a las divergencias y convergencias de la red de significantes que están en relación al concepto de calidad. En esta operación, se hizo hincapié en las interpretaciones contradictorias que giran en torno al concepto de calidad.

Las tres operaciones presentadas anteriormente tuvieron en cuenta el análisis de los 4 signos propuestos por Magariños de Morentin (2004). Este análisis hace referencia a la intención de comprender un fenómeno desde la perspectiva semiótica desde la producción, interpretación, contrastación y superación del significado. Por esta razón el trabajo realizado tuvo en cuenta el siguiente proceso:

1. Descripción del proceso de producción de la significación otorgada al concepto de calidad en los textos analizados.

2. Explicación del proceso de interpretación de la significación otorgada al concepto de calidad en la educación para el estado y las instituciones de educación superior, en los documentos analizados.

3. Reconocimiento del proceso de contrastación de la significación otorgada al concepto 
de calidad en la educación para el estado y las instituciones de educación superior.

4. Análisis del proceso de superación de la significación otorgada al concepto de calidad en la educación para el estado y las instituciones de educación superior con relación a los datos obtenidos.

Cabe resaltar que el tratamiento de los datos a partir de los 4 signos se ajusta a cada una de las operaciones presentadas.

Operación 1 = Descripción del proceso de producción.

Operación 2 = Explicación del proceso de interpretación.

Operación 3 = Reconocimiento del proceso de contrastación.

Y con respecto al 4to signo (análisis del proceso de superación de la significación), éste se llevó a cabo en la parte concluyente del trabajo donde se hizo una reflexión del proceso de significación de la calidad y su impacto en el contexto educativo colombiano.

Después de explicar la parte metodológica me permito presentar los hallazgos y resultados encontrados hasta el momento, dado que el proceso de investigación sigue en curso. Los resultados obtenidos en esta fase permiten aclarar que definitivamente existe un problema de significación en el concepto de calidad. Esta situación se da porque no existe un ajuste de las condiciones de satisfacción entre las políticas educativas y las políticas institucionales. Este problema se evidenció en la no convergencia de las intencionalidades entre el estado y la universidad. La situación es que el concepto de calidad para el estado colombiano está en función de una significación netamente económica y utilitarista, mientas que las instituciones significan la calidad a partir de la generación de nuevos conocimientos que posibiliten el desarrollo del país y el estudiante. Al no existir este ajuste en las transformaciones del significado se crea una brecha amplia que imposibilita el desarrollo de una política clara de calidad.

Por otra parte, se encontró que las pruebas para la estandarización del conocimiento en Colombia son un fin mas no un medio. Es decir, que las pruebas, según la significancia otorgada a la misma, son las únicas que pueden determinar la calidad. Este resultado es muy interesante e importante ya que la significación de las pruebas saber pro sí poseen un ajuste en la condición de satisfacción entre el sistema de signos del estado y el sistema de signos de las instituciones de educación. A raíz de esto, podemos inferir que la significancia tiene ristres de convergencia y divergencia, lo cual aumenta la necesidad de implementar la metodología semiótica en los estudios relacionados con los procesos de educación.

La investigación también demostró que la diferencia en los sistemas de signos también se debe a las condiciones intersubjetivas como lo pueden ser la cultura y las condiciones propias de los sujetos. Es decir que el proceso de generación del significado influye mucho las condiciones intersubjetivas las cuales deben ser estudiadas en los procesos de significación educativa. Esto se encontró en las divergencias presentadas en las políticas institucionales las cuales tenían relación a la enseñanza de la lectura y la escritura. No obstante, es necesario aclarar que en todas se ve a la lectura y la escritura como estrategia para el mejoramiento de las pruebas para el aseguramiento de la Calidad. 


\section{CONCLUSIONES}

Como último resultado encontramos una posible hipótesis con relación a las diferencias interpretativas que giran en torno al concepto de calidad. Esta hipótesis plantea la posibilidad de ver el problema en cuestión como el resultado de un fallo en la ética discursiva del estado y las instituciones educativas. Para aclarar esto se toma referencia a la teoría de Apel. Según Apel (2002), la función comunicativa requiere de una gran comprensión a partir de los diversos fenómenos que suceden el mismo. Para Apel (2008), la única forma de comprensión del lenguaje es mediante la racionalidad consensual comunicativa. En este sentido cualquier acto comunicativo debe superar los componentes intencionales y de verdad. Para Apel (2008), continuando la línea de Habermas, el componente de lenguaje refiere a unas condiciones de adaptalividad en las acciones. Esta idea se fundamente en su postura de una filosofía trascendental del lenguaje "en el sentido de una reconstrucción crítica y una transformación de una prima philosophia fundamentada por Kant, que pretende responder la pregunta por las condiciones de posibilidad de la argumentación válida (Apel, K. 2008. p. 115). En este sentido, Apel utiliza los tres funciones del lenguaje de Bühler (expositiva, expresiva y apelativa) a partir de las tres dimensiones del mundo (mundo de los objetos, mundo social y mundo interior). Con esta integración Apel (2008) menciona la necesidad de entender los actos comunicativos como fenómenos que se integran en los criterios de valides, o de argumentación válida. Con dicha perspectiva, el autor presenta una formula tripartita para saber si se consolida la validez, tomando como ejemplo los actos ilocucionarios . La fórmula tripartita de Apel (2008) retomando a Habermas es la siguiente:

"El significado de los actos ilocusionarios" se puede ver a partir de las "tres dimensiones de posible validez":

1. La pretensión de verdad referida al mundo objetivo, cuyo portadores son las preposiciones afirmadas de los actos de habla constatativos o asertóricos .

2. La pretensión de rectitud de los actos de habla en cuanto actos comunicativos, con referencia a las normas (jurídicas y morales) del mundo social, los cuales plantean una pretensión frente a los destinatarios.

3. La pretensión de veracidad o sinceridad de los actos de hable en cuanto automanifestación expresiva que exponen algo del mundo interior, subjetivo del hablante.

Teniendo claro estas tres configuraciones se puede decir que el discurso, para que sea válido, debe unir o debe integrase en las tres dimensiones. En síntesis, a la hora de analizar un acto comunicativo se debe tener en cuenta el criterio de validez que se configura en las tres dimensiones anteriormente expuestas.

Con esta explicación, y correlacionándola los resultados obtenidos, se puede decir que existe un conflicto ético discursivo de la calidad en la educación en Colombia. No obstante, esta hipótesis se sigue estudiando.

En conclusión, el análisis semiótico realizado al concepto de calidad en Colombia, permitió ampliar los enfoques investigativos utilizados hasta el momento en la educación. Así mismo, la investigación desarrollada permite ver nuevos modelos paradigmáticos que apo- 
yarían al mejoramiento de los procesos formativos en Colombia y el mundo. Para terminar vale la pena recordar que la consolidación del conocimiento se da mediante un transformación semántica de los signos presentes en las interacciones. Por esta razón, es necesario ampliar este tipo de aportes para mejorar y ampliar el discernimiento que se tiene hasta el momento del fenómeno educativo.

\section{REFERENCIAS}

Apel, K. (1991). Teoría de la verdad y ética del discurso. Barcelona: Paidos. Apel, K. (2008). Semiótica Filosófica. Madrid: Prometeo.

Apel, K. (2002). Semiótica trascendental y filosofía primera. Madrid: Editorial Síntesis.

Beuchot, M. (2004). La semiótica. Teorías del signo y del lenguaje en la historia. MéxicoD.F.: FCE.

Cassirer, E. (2003). Filosofía de las formas simbólicas. México: FCE.

Consejería para la Modernización del Estado. (1994). Colombia: al filo de la oportunidad.

Greimas, A.J. y Courtés, J. (1991). Semiótica. Diccionario razonado de la teoría del lenguaje. Madrid: Editorial Gredos.

Kondrátov, A.M. (1973). Del Sonido al Signo. Buenos Aires: Paidos.

Informe conjunto Misión Ciencia, Educación y Desarrollo. Bogotá: Colciencias. Eco, U. (2013). Interpretación y Sobreinterpretación. Tres Cantos: AKAL.

Eco, U. (1977). Tratado General de Semiótica. Madrid: Lumen

. (2013). Los límites de la interpretación. Barcelona: De Bolsillo.

. (2013). Interpretación y Sobreinterpretación. Tres Cantos: AKAL.

Espinosa, E. Pérez, R. Blackaller, J. (1995). Algunas consideraciones en torno a la problemática de la implantación de los modelos de calidad total en las pequeñas y medianas empresas mexicanas. En la Revista "Gestión y Estrategia" No. 8 Julio- Diciembre.

Estrada, L. (1999). Hacia un modelo de evaluación de la calidad de instituciones de educación superior. OEI - Ediciones - Revista Iberoamericana de Educación - Número 21.

Gabalán, J. (2007). ECAES: una visión sistémica. El Hombre y la Máquina No. 29, Julio - Diciembre de 2007.

Jakobson, R. (2012). El marco del lenguaje. México: FCE. Luhmann, N. (2005). Poder. Barcelona: Anthropos.

Magariños de Morentín, J. (2008): La semiótica de los bordes. Apuntes para una metodología semiótica. Córdoba: Comunic-Arte.

. (1998). Manual operativo para la elaboración de "definiciones contextuales" y "redes contrastantes", en Signa. Revista de la Asociación Española de Semiótica, no 7; 233253.

. (1999). Los Mundos Semióticos Posibles en la Investigación Social/Possible Semiotic Worlds in Social Research, en Archivos de la Universidad Nacional de La Plata, Vol. I, Núm. 1. Octubre.

. (2004). Los 4 signos. Diseño de las operaciones fundamentales en metodología semiótica, en Razón y Palabra 38: Semiótica e Informática, una nueva alianza, Prime- 
ra Revista Electrónica de América Latina especializada en Comunicación; Tecnológico de Monterrey.

. (2007). La semiótica de los bordes, en Significación y negatividad, Tópicos del Seminario № 18: ps. 97-112. Benemérita Universidad Autónoma de Puebla, Seminario de Estudios de la Significación; Puebla, México . (1993). La semiótica de enunciados. La Plata: IICS, Universidad Nacional de la Plata Peirce, Ch. (1970), Deducción, inducción e hipótesis. Argentina: Aguilar. - (1974), La ciencia de la semiótica. Buenos Aires: Ediciones Nueva Visión. . (2007). La lógica considerada como semiótica. Madrid: Biblioteca Nueva. . (1987), Obra lógico semiótica. Taurus: Madrid.

Piaget, J. (1978). Memoria e Inteligencia. Buenos Aires: El Ateneo. Morris, Ch. (1962). Signos, lenguaje y conducta. Buenos Aires: Lozada. Searle, J. (1994). Actos del habla. Barcelona: Planeta de Agostini. . (1999). Intencionalidad: un ensayo de la filosofía de la mente. Barcelona: Altaya.

Servat, B. (1996). "Participación y eficacia en la escuela: estudios empíricos". Boletín de Investigación Educacional N' 1 1, pp. 245-285. Facultad de Educación. PUC. Santiago de Chile.

Stables, A. \& Semesky, I. (2014). Edusemiotics. Semiotic philosophy as educational foundation. New York: Routledge. 